\title{
Core-Substituted Naphthalene Diimides: Influence of Substituent Conformation on Strong Visible Absorption
}

Samuel Quinn, E. Stephen Davies, Constance R. Pfeiffer, William Lewis, Jonathan McMaster and Neil R. Champness*[a]

School of Chemistry, University of Nottingham, Nottingham, NG7 2RD. E-mail: Neil.Champness@nottingham.ac.uk

Abstract: Substitution of the aromatic core of naphthalene diimide (NDI) chromophores by morpholine leads to molecules with strong absorbance in the visible spectrum. The shift of absorption maxima to lower energy is determined not only by the degree of substitution but also by the relative conformation and orientation of the tertiary amine with respect to the plane of the NDI.

1,4,5,8-Naphthalenetetracarboxylic acid diimides (NDIs) are a class of dye molecules that have been investigated extensively because of their applications in materials and supramolecular chemistry. The robust nature of the compounds and their attractive photochemical properties have led to their use in various applications ${ }^{[1-3]}$ including organic electronics ${ }^{[4-6]}$ and photovoltaic devices. ${ }^{[7-14]}$ With regard to supramolecular chemistry, NDIs have been utilised in anion recognition ${ }^{[15-17]}$ and the syntheses of metal-organic supramolecular structures. ${ }^{[18]}$ Unsubstituted NDIs are typically lightly coloured, often pale yellow, and functionalisation of the naphthalene core is required to facilitate strong absorption in the visible region by these dye molecules. For such systems to be employed as electron acceptors in organic photovoltaic devices, or indeed in other applications as dyes, it is vital to fully appreciate the factors that determine the absorption properties of this important class of molecule. One method to achieve this is through systematic substitution of the NDI core.

Functionalisation of NDIs can be readily achieved at the imide position and such substitution has been successfully employed to manipulate the solubility and aggregation properties of these chromophores. Substitution of the naphthalene core is more synthetically demanding but has been successfully achieved and typically leads to a more direct modification of the absorption properties. ${ }^{[19,20]}$ Herein we have chosen to investigate the effect of tertiary amine substitution on the absorption properties of these important dye molecules. We demonstrate that the absorption properties are influenced not only by the degree of amine substitution but also by the orientation of the amine, and in particular the amine lone pair, relative to the NDI core.
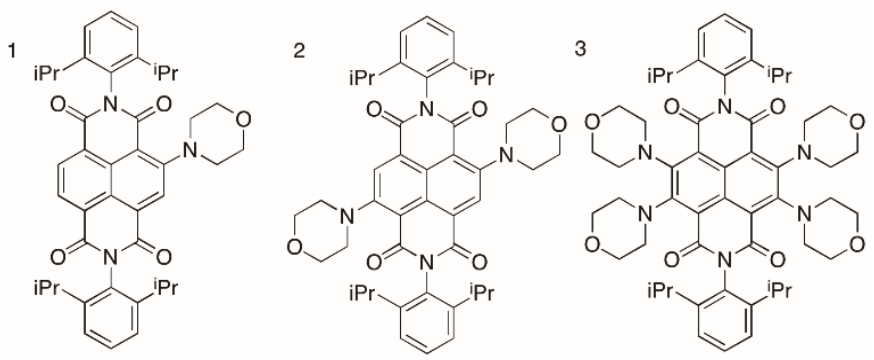

Figure 1. Mono- (1), di- (2) and tetra- (3) morpholine-substituted NDIs reported in this study. 
Mono- (1), di- (2) and tetra- (3) substituted NDIs (Figure 1) were synthesised by morpholine substitution of the corresponding brominated NDI. The mono- and di-bromo NDIs were prepared by literature procedures, ${ }^{[21]}$ isolated and reacted with morpholine in DMF solution to yield $\mathbf{1}$ and $\mathbf{2}$, in $91 \%$ and $81 \%$ yield respectively. For 3 , the tetra-bromo NDI was not isolated prior to the reaction with morpholine due to increased sensitivity of this intermediate with respect to decomposition, see SI for details. $\mathbf{3}$ was formed in considerably lower yield (3\%), in comparison to $\mathbf{1}$ and $\mathbf{2}$, due to debromination of the starting material under the reaction conditions resulting in formation of $\mathbf{1}$ and $\mathbf{2}$ as major products from the reaction. Reduced yields for reactions of tetra-bromo NDI with amines have been previously discussed. ${ }^{[22]}$

a)

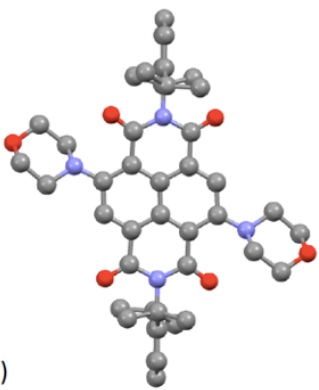

c)

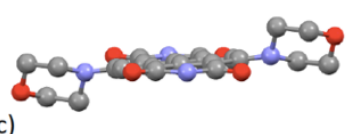

b)

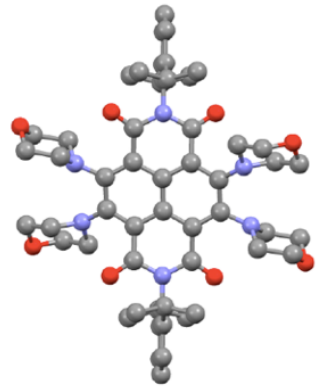

d)

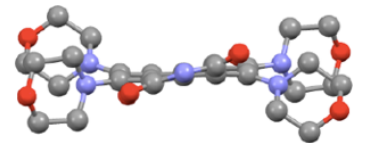

Figure 2. Single crystal structures of $(a, c) 2$ and $(b, d) 3$. The different orientations of the morpholine substituents in $\mathbf{2}$ and $\mathbf{3}$, with respect to the NDI plane, is clearly seen in c) and d) respectively. Carbon - grey, nitrogen- blue, oxygen- red. Hydrogen atoms and, in c) and d), the imide substituents are omitted for clarity.

Single crystals of 1-3 were grown by vapour diffusion of $\mathrm{MeOH}(\mathbf{1}, \mathbf{2})$ or hexane $(3)$ into $\mathrm{CHCl}_{3}$ solutions of the compounds. ${ }^{[23]}$ The structures not only confirmed the chemical composition of the three compounds but importantly showed the conformational arrangements of the three species, in particular the relative orientation of the morpholine substituents with respect to the NDI core (Figures 2 and S1) in the solid state. In order to evaluate the conformational arrangements in 1-3 the angle adopted between the least squares planes formed by the naphthalene diimide and the plane formed by the $\mathrm{N}$ atom and three adjacent carbon atoms of each morpholine substituent was measured (two molecules are observed in the crystallographic asymmetric unit for $\mathbf{1}$ and $\mathbf{2}$ ). The angles between the planes are similar in $\mathbf{1}\left(38.2^{\circ}\right.$ and $\left.42.2^{\circ}\right)$ and $\mathbf{2}\left(42.0^{\circ}\right.$ or $\left.46.2^{\circ}\right)$. However, in the case of 3 this angle was found to be $48.7^{\circ}$ for two of the appendages and $79.3^{\circ}$ for the other two substituents (nearly perpendicular to the naphthalene diimide plane). Thus, two of the morpholine units in $\mathbf{3}$ adopt a similar conformation to those in $\mathbf{1}$ and $\mathbf{2}$ whereas the remaining morpholine groups adopt a near perpendicular orientation with respect to the NDI plane in the solid-state structure of $\mathbf{3}$.

It is apparent from the highly coloured solutions formed by 1-3 that morpholine substitution has a significant effect on the optical properties of NDI, as is expected for the incorporation of electron donating substituents like amines. ${ }^{[19,20,24]}$ A considerable shift of the absorption maxima into the visible is observed upon addition of morpholine substituents to the NDI unit. Whereas mono-bromo NDI is pale yellow in $\mathrm{CHCl}_{3}$ solution, 1 is pink $\left(\lambda_{\max }=541 \mathrm{~nm}\right)$ and 2 is blue $\left(\lambda_{\max }=602 \mathrm{~nm}\right)$ (Figure 3, 4). 
Surprisingly 3 was found to be a similar colour $\left(\lambda_{\max }=550 \mathrm{~nm}\right)$ to 1 despite the incorporation of three additional morpholine substituents. The bathochromic shift in the absorption maxima upon the introduction of additional morpholine groups in $\mathbf{1}$ and $\mathbf{2}$ is consistent with our previous observations with related PDI analogues ${ }^{[25,26]}$ and with related amino-functionalised NDIs. ${ }^{[19,20,24]}$ Surprisingly, the addition of four morpholine groups onto the naphthalene core, 3, results in a similar UV-visible spectroscopic profile to that for $\mathbf{1}$, and is in stark contrast to previously related examples of tetraamino-functionalised NDIs. ${ }^{[24]}$

Cyclic voltammetry and UV-visible spectroelectrochemistry was conducted to further investigate the influence of morpholine substitution upon the optical properties of 1-3. Two reduction processes are observed for 1-3 (Figure 5 and SI for further details), consistent with previously reported $\mathrm{NDIs},{ }^{[20]}$ with $\mathrm{E}_{1 / 2}$ values of $-1.10,-1.56 \mathrm{~V}(\mathbf{1}),-1.15,-1.61 \mathrm{~V}(\mathbf{2})$ and $-1.30,-1.68 \mathrm{~V}(\mathbf{3}) \mathrm{vs} . \mathrm{Fc} / \mathrm{Fc}^{+}$. EPR spectra for the electrogenerated monoanions were recorded confirming the formation on radical anions upon reduction (see $\mathrm{SI}$ ). We note that an increase in the number of electron-donating morpholine substituents results in a systematic shift of reduction potential to more negative values, which is entirely consistent with previous reports of amino-functionalised NDIs. ${ }^{[19,20,24]}$ For $\mathbf{3}$ an additional redox process is observed at $c a .-1.0 \mathrm{~V}$ on the return wave consistent with the formation of an electro-generated by-product following the second NDI reduction process. The compounds exhibit complex oxidative processes (see $\mathrm{SI}$ ) which were not probed further by spectroelectrochemistry.

In situ spectroelectrochemical methods were employed to probe the UV-visible spectroscopic changes that accompany the one- and two-electron reduction processes of $\mathbf{1}$ and $\mathbf{2}$ (Figure 6 and $\mathrm{SI}$ ). Such studies are unusual for NDI-containing species. ${ }^{[27,28]}$ In the case of $\mathbf{3}$ only the first reduction was assessed due to the instability of the dianion, as noted from cyclic voltammetry. It is notable that although absorption maxima for 1-3 do not follow a linear trend, a hypsochromic trend being observed between $\mathbf{2}$ and $\mathbf{3}$, this same pattern is not observed for the mono-reduced species. Evaluation of the absorption maxima for each monoanion reveals a bathochromic shift from [1] $]^{-}\left(\lambda_{\max }=495 \mathrm{~nm}\right)$ to [2] $\left(\lambda_{\max }=505 \mathrm{~nm}\right)$ to [3] $]^{-}\left(\lambda_{\max }=526 \mathrm{~nm}\right)$. (See SI for full details of spectroelectrochemistry and UV-vis data for reduced species).

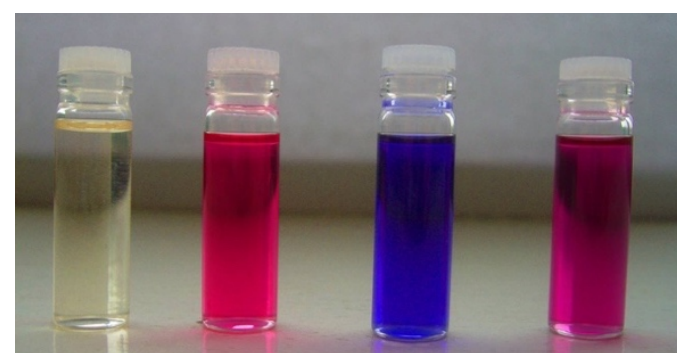

Figure 3. Solutions of $\mathrm{NDI}$ species in $\mathrm{CHCl}_{3}$ solution. From left to right $\mathrm{Br}-\mathrm{NDI}, \mathbf{1}, \mathbf{2}, \mathbf{3}$. 


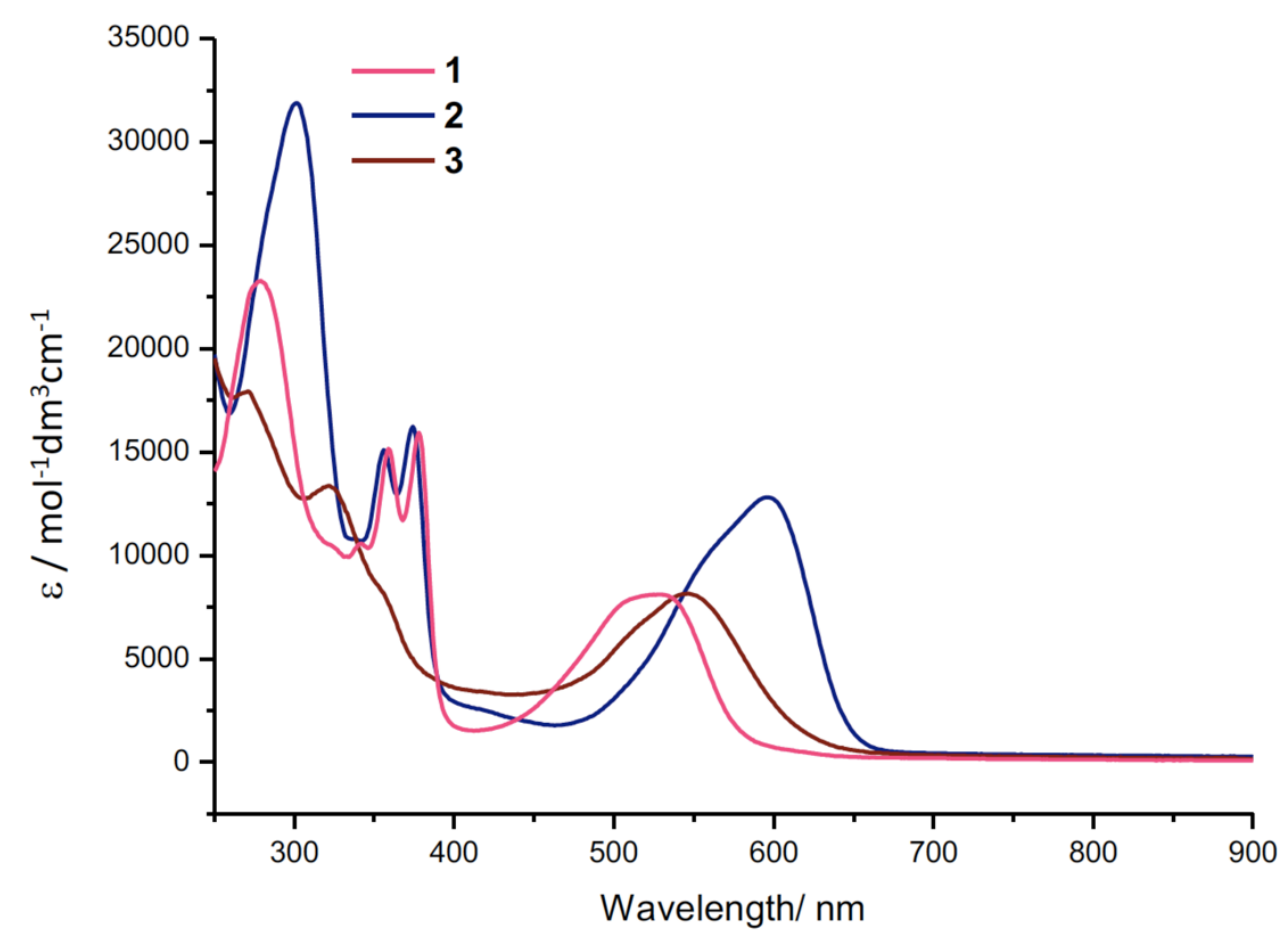

Figure 4. UV/vis spectra of $\mathbf{1}$ (pink), $\mathbf{2}$ (blue), and $\mathbf{3}$ (purple) in $\mathrm{CH}_{2} \mathrm{Cl}_{2}$ solution.

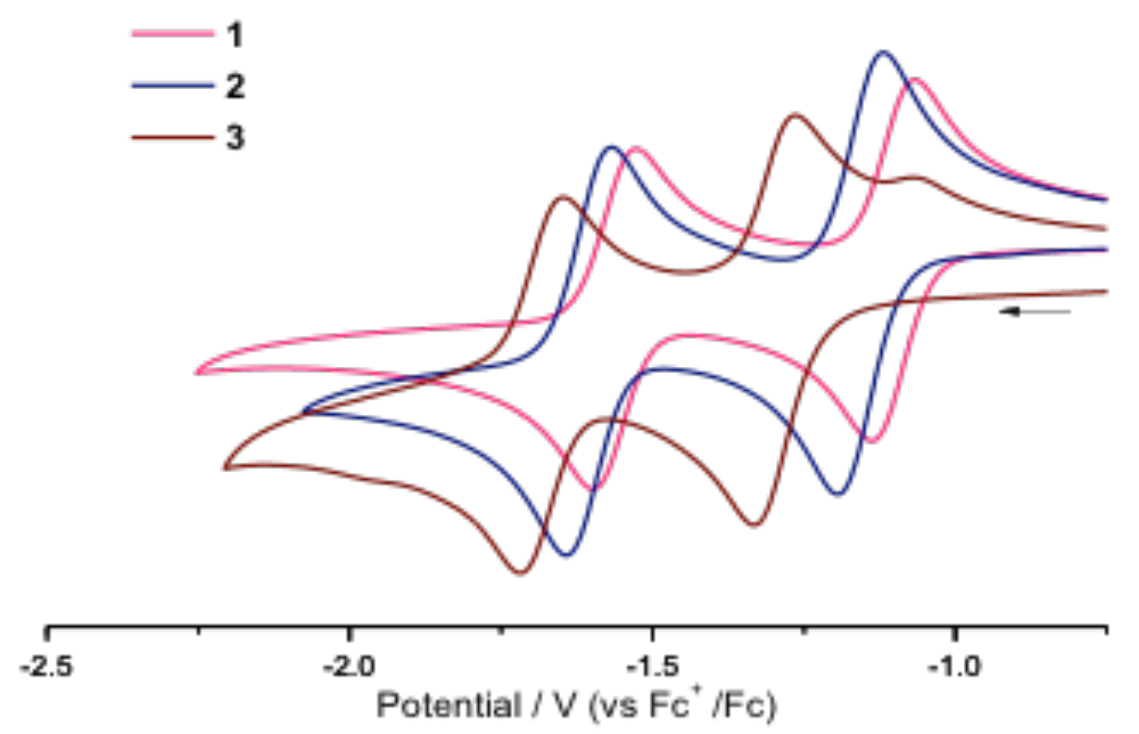

Figure 5. Cyclic voltammograms of $\mathbf{1}$ (pink), $\mathbf{2}$ (blue) and purple (3) recorded in $\mathrm{CH}_{2} \mathrm{Cl}_{2}$ containing $\left[\mathrm{Bu}_{4} \mathrm{~N}\right]\left[\mathrm{BF}_{4}\right](0.4 \mathrm{M})$.

It is interesting to note that for this series of compounds an interesting contrast is observed between the trends in reduction potential and absorption maxima for 1-3. Thus, the reduction potentials follow the trend $\mathbf{1}>\mathbf{2}>\mathbf{3}$ whereas the absorption maxima $(\mathrm{nm})$ follow the trend $\mathbf{2}>\mathbf{3}>\mathbf{1}$. In order to investigate these trends further we performed density functional theory (DFT) and timedependent DFT calculations on geometry optimized models of 1-3 in a dichloromethane solvent field 
in order to evaluate the influence of morpholine substitution on the electrochemical and UV-visible spectroscopic properties of the compounds (see SI for full details).
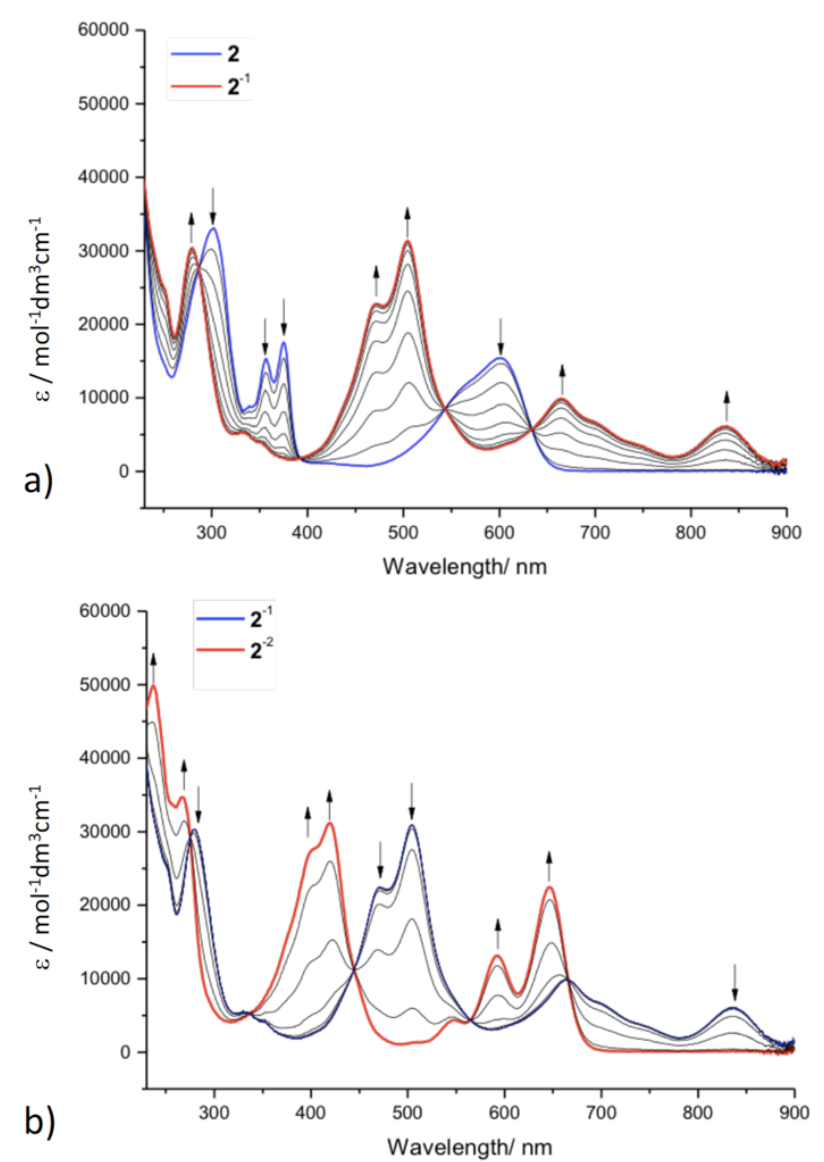

Figure 6. UV-visible spectra of $\mathbf{2 / 2} / \mathbf{2}^{2-}$ recorded in $\mathrm{CH}_{2} \mathrm{Cl}_{2}$ containing $\left[\mathrm{Bu}_{4} \mathrm{~N}\right]\left[\mathrm{BF}_{4}\right](0.4 \mathrm{M})$ using spectroelectrochemical methods for $\mathbf{2}$ at $273 \mathrm{~K}$ showing a) the inter-conversion of $\mathbf{2}$ (blue) to $\mathbf{2}^{-}$(red); and b) $\mathbf{2}^{-}$(blue) to $\mathbf{2}^{\mathbf{2 -}}$ (red). Arrows indicate the progress of the reduction.

The angles between the morpholine units and the NDI planes in the calculated DFT geometry optimised structures of 1 and $\mathbf{2}\left(40.5\right.$ and $43.3^{\circ}$, respectively, Tables S5 and S6) are similar to those for the experimental structures $\left(38.2^{\circ}\right.$ or $42.2^{\circ}$ for 1 , and $42.0^{\circ}$ or $46.2^{\circ}$ for 2 ). In contrast the calculated angles for $3\left(54.5,61.3,61.5\right.$ and $68.1^{\circ}$, Table 57$)$ do not reproduce the near perpendicular orientation for two of the morpholine units in the experimental solid state structure of 3 . Furthermore the greater angles between the morpholine units and the NDI plane in $\mathbf{3}$ relative to those of $\mathbf{1}$ and $\mathbf{2}$ appears to be in line with greater steric congestion about the NDI bay area in $\mathbf{3}$ that arises from the substitutions of four morpholine group to the NDI unit. 


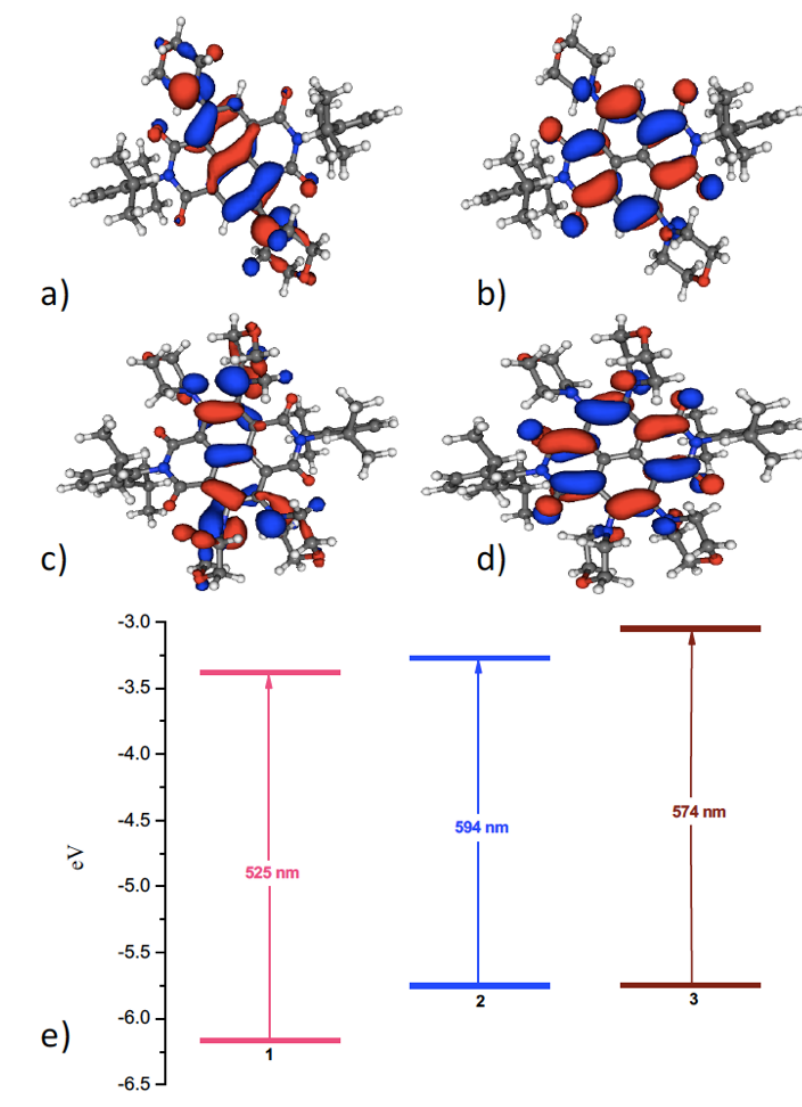

Figure 7. DFT calculated HOMOs and LUMOs for 2 and 3. Compound 2: a) HOMO, b) LUMO. Compound 3 : c) HOMO, d) LUMO. e) Comparison of HOMO-LUMO energy gaps and calculated values for $\lambda_{\max }$ for 1-3.

Nevertheless, the range of angles between the morpholine units and the NDI plane in the experimental and calculated structures of $\mathbf{3}$ suggests that the morpholine units adopt orientations which are most likely limited by the relative proximity and conformations of the adjacent morpholine units. The calculated compositions of the HOMO in 1-3 (Tables S5-7, Figures 7 and S2) show that these are NDI- and morpholine-based with contributions that vary between 50.2, 45.8 and 32.8 (\%NDI) and 49.3, 54.0 and 66.9 (\%morpholine), for 1-3 respectively. In contrast the LUMOs in 1-3 are essentially localized on the NDI units with contributions of 94.4, 92.1 and 87.0 (\%NDI) and 5.3, 7.6 and 12.5 (\%morpholine). The energies of the HOMO and LUMO in 1-3 follow the trend $\mathbf{3}>\mathbf{2}>\mathbf{1}$ which is in line with the incorporation of one, two and four morpholine units in 1, $\mathbf{2}$ and $\mathbf{3}$, respectively. However, we note that the HOMO in $\mathbf{3}$ shows only a marginal increase in energy relative to its counterpart in $\mathbf{2}$ (Figure 7, Table S1), which may relate to the significantly different orientations of the morpholine units relative to the NDI plane in $\mathbf{3}$ when compared to those in $\mathbf{1}$ and $\mathbf{2}$. This effect is not apparent in the trend in energies for the LUMOs presumably because of the significantly lower contributions from the morpholine units in these orbitals. The calculated energies of the LUMOs follow the order $3>2>1$ and mirror the measured reduction potentials $\mathbf{1}>\mathbf{2}>\mathbf{3}$. Similarly, the calculated trend in $\lambda_{\max }(\mathrm{nm}) \mathbf{2}$ > 3 > 1 (Figure 7, Tables S2-S4) follows that found experimentally and which can be rationalised qualitatively in terms of the relative energies of the HOMO and LUMO orbitals in each 1-3 discussed above. Thus, the apparent hypsochromic shift in $\lambda_{\max }$ from $\mathbf{2}$ to $\mathbf{3}$ may result from a greater difference in HOMO and LUMO energies for 3; a consequence of similar HOMO energies for $\mathbf{2}$ and $\mathbf{3}$ that may 
reflect the different orientations of the morpholine units relative to the NDI plane in $\mathbf{3}$ when compared to those in $\mathbf{2}$.

In conclusion, we have demonstrated that it is readily possible to functionalise NDIs with amine functionalities that give rise to highly coloured dye molecules. Whilst the expected shift in redox potentials to more negative values follows the increase in number of electron donating amine substituents in 1, $\mathbf{2}$ and 3, (one, two and four respectively), the resulting absorption properties are far from straightforward. In contrast to previously reported examples of amino-functionalised NDIs, tetra-substitution by morpholine in $\mathbf{3}$ does not give the expected hypsochromic shift in the absorption maxima in comparison to the di-substituted species, $\mathbf{2}$. Through a combination of single crystal X-ray diffraction and time dependent DFT calculations we conclude that the orientation of the morpholine substituents with respect to the naphthalene plane are an important factor in controlling the properties of functionalised NDIs. Thus, it can be concluded that steric and conformational factors are significant, not just electronic effects, when designing sterically congested functionalised NDIs.

\section{Experimental Section}

Full synthetic details, cyclic voltammetry and UV-vis spectroelectrochemistry data, EPR spectra of monoanions, DFT Geometry Optimized Structures and figures of the single crystal structure of $\mathbf{1}$ are available in the Supporting Information.

\section{Acknowledgements}

NRC gratefully acknowledges a Royal Society Wolfson Merit Award.

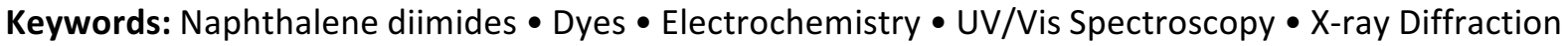

[1] S. V. Bhosale, C. H. Jani, S. J. Langford, Chem. Soc. Rev. 2008, 37, 331-342.

[2] S.-L. Suraru, F. Würthner, Angew. Chem. Int. Ed. 2014, 53, 7428-7448.

[3] M. Al Kobaisi, S.V. Bhosale, K. Latham, A.M. Raynor, S.V. Bhosale Chem. Rev., 2016, 116, 11685-11796.

[4] M., Sommer, J. Mater. Chem. C 2014, 2, 3088-3098.

[5] X.; Zhan, A. Facchetti, S. Barlow, T. J. Marks, M. A. Ratner, M. R. Wasielewski, S. R. Marder, Adv. Mater. 2011, 23, 268-284.

[6] Y. Zhao, Y. Guo, Y. Liu, Adv. Mater. 2013, 25, 5372-5391.

[7] J. G. Laquindanum, H. E. Katz, A. Dodabalapur, A. J. Lovinger, J. Am. Chem. Soc. 1996, 118, 11331-11332.

[8] Z. Chen, Y. Zheng, H. Yan, A. Facchetti, J. Am. Chem. Soc. 2009, 131, 8-9.

[9] M. Borgström, N. Shaikh, O. Johansson, M. F. Anderlund, S. Styring, B. Åkermark, A. Magnuson, L. Hammarström, J. Am. Chem. Soc. 2005, 127, 17504-17515.

[10] F. Chaignon, F. Buchet, E. Blart, M. Falkenstrom, L. Hammarstrom, F. Odobel, New J. Chem. 2009, 33, 408-416.

[11] E. lengo, G.D. Pantos, J. K. M. Sanders, M. Orlandi, C. Chiorboli, S. Fracasso, F. Scandola, Chem. Sci. 2011, 2, 676-685.

[12] V. L. Gunderson, A. L. Smeigh, C. H. Kim, D. T. Co, M. R. Wasielewski, J. Am. Chem. Soc. 2012, $134,4363-4372$.

[13] N. Sakai, R. Bhosale, D. Emery, J. Mareda, S. Matile, J. Am. Chem. Soc. 2010, 132, 6923-6925.

[14] A. Rananaware, D.D. La, S.M. Jackson, S.V. Bhosale, RSC Adv., 2016, 6, 16250- 16255. 
[15] S. T. Schneebeli, M. Frasconi, Z. Liu, Y. Wu, D. M. Gardner, N. L. Strutt, C. Cheng, R. Carmieli, M. R. Wasielewski, J. F. Stoddart, Angew. Chem. Int. Ed. 2013, 52, 13100-13104.

[16] R. E. Dawson, A. Hennig, D. P. Weimann, D. Emery, V. Ravikumar, J. Montenegro, T. Takeuchi, S. Gabutti, M. Mayor, J. Mareda, C. A. Schalley, S. Matile, Nat. Chem. 2010, 2, 533-538.

[17] S. Guha, S. Saha, J. Am. Chem. Soc. 2010, 132, 17674-17677.

[18] M. Pan, X.-M. Lin, G.-B. Li, C.-Y. Su, Coord. Chem. Rev. 2011, 255, 1921-1936.

[19] R. Bhosale, A. Perez-Velasco, V. Ravikumar, R. S. K. Kishore, O. Kel, A. Gomez-Casado, P. Jonkheijm, J. Huskens, P. Maroni, M. Borkovec, T. Sawada, E. Vauthey, N. Saka, S. Matile, Angew. Chem. Int. Ed., 2009, 48, 6461-6464.

[20] R. S. K. Kishore, O. Kel, N. Banerji, D. Emery, G. Bollot, J. Mareda, A. Gomez-Casado, P. Jonkheijm, J. Huskens, P. Maroni, M. Borkovec, E. Vauthey, N. Sakai, S. Matile, J. Am. Chem. Soc., 2009, 131, 11106-11116.

[21] Y. V. Suseela, M. Sasikumar, T. Govindaraju, Tetrahedron Lett., 2013, 54, 6314-6318.

[22] S.V. Bhosale, C. Jani, C. H. Lalander, S.J. Langford, Chem. Commun., 2010, 46, 973-975.

[23] CCDC 1523090, 1523091 and 1523092 contain the supplementary crystallographic data for this paper for 1, $\mathbf{2}$ and $\mathbf{3}$ respectively. These data can be obtained free of charge from The Cambridge Crystallographic Data Centre via www.ccdc.cam.ac.uk/data_request/cif.

[24] C. Roger, F. Wurthner, J. Org. Chem., 2007, 72, 8070-8075.

[25] G. Goretzki, E. S. Davies, S. P. Argent, W. Z. Alsindi, A. J. Blake, J. E. Warren, J. McMaster, N. R. Champness, J. Org. Chem., 2008, 73, 8808-8814.

[26] B.A. Llewellyn, E.S. Davies, C.R. Pfeiffer, M. Cooper, W. Lewis, N.R. Champness, Chem. Commun., 2016, 52, 2099-2102.

[27] M. Jaggi, B. Schmid, S-X. Liu, S.V. Bhosale, S. Rivadehi, S.J. Langford, S. Decurtins, Tetrahedron, 2011, 67, 7231-7235.

[28] H. Jia, B. Schmid, S-X. Liu, M. Jaggi, P. Monbaron, S.V. Bhosale, S. Rivadehi, S.J. Langford, L. Sanguinet, E. Levillain, M.E. El-Khouly, Y. Morita, S. Fukuzumi, S. Decurtins, ChemPhysChem, 2012, 13, 3370-3382. 